\title{
BASES PARA EL CONOCIMIENTO DE LA PRESENCIA TIWANAKU EN EL VALLE DEL CAPLINA, TACNA
}

\author{
Carlos Vela Velarde ${ }^{1}$
}

\author{
R E S U M E N
}

La presencia tiwanaku en el valle del Caplina es consecuencia del proceso de expansión de Tiwanaku (aprox. 800-900 d.C.). Este proceso logró cohesionar los pueblos del sur del Perii y norte de Chile actuales con la zona de Bolivia. El valle del Caplina recibio el influjo del Imperio Tiwanaku, arqueologicamente se describen materiales de fabricación loca. La presencia tiwanaku en el valle del Caplina (Tacna) es considerada una más pues Tacna en su historia ha recibido varias presencias culturales que han dejado su 'impronta' en las identidades y en las mentalidades de su población.

\section{A B S T R A C T}

The tiwanaku presence in the Caplina valley is consequence of the process of Tiwanaku expansionism (around 800-900 a.C.), this process brought as a result the gathering of towns from the south of Perú, north of Chile and part of Bolivia. The Caplina valley received the influence of the tiwanaku empire; materials of local manufacturing are archaelogically described.

The tiwanaku presence in the Caplina valley (Tacna) is considered as one more, since Tacna throughout its history has received several presences from others cultures that have left their 'improntus' in the indetities and mentalities of its population.

\section{INTRODUCCIÓN}

En estos tiempos que corren, ricos en unidades socio-culturales conflictivas de premodernidad, modernidad y postmodernidad, la arqueología resulta una ciencia absolutamente vanguardista.

Las metodologias teóricas y prácticas de la arqueología nos permiten indagar el pasado, rescatar las huellas de entonces y convertirlas en presente perpetuo, de cara a un futuro que, de ese modo, deviene en más certero y probablemente menos fortuito. La arqueología no sólo es capaz de rescatar la prehistoria, sino que, plausiblemente, puede reubicarse en el presente, para efectos de producción y la identidad, objetos, simbolos e infraestructuras, que habiendo cumplido una función en su momento,

1. Arqueólogo. bien pueden volver a hacerlo, reforzando valores, concepciones y asi en el proceso de 'globalización', la arqueología contribuye fortaleciendo identidades, pues no podemos renunciar a nuestro derecho al desarrollo, pero tenemos que participar con personalidad y conviviendo con nuestra historia y nuestras tradiciones.

La arqueologia tiene que ver con el desarrollo y el futuro de progreso de cualquier sociedad.

Está claro, por ejemplo, que las tecnologias de punta resultan exclusivistas, caras y hasta en ocasiones desfasadas de las prioridades de las sociedades dependientes y oprimidas. En su lugar han cobrado notorio valor e inusitada vigencia las denominadas tecnologías tradicionales, que pueden proceder del quehacer arqueológico.

La arqueologia permite a los pueblos convivir con su historia y perpetuar sus identidades y sus 
procesos vitales, de forma que venzamos al tiempo, el mismo que jamás pasa por el mismo sitio dos veces, y extraerle más bien la posibilidad de que se detenga en nosotros, en el imaginario colectivo o en la conciencia social.

La arqueología, pues, nos permite una reinvención de nosotros como sociedad y como cultura. Estas consideraciones conceptuales nos han guiado en todo momento. Hace ya un buen tiempo, nuestras preocupaciones arqueológicas se centran en TIWANAKU, principalmente en su presencia en el valle del Caplina, Tacna.

Actualmente la zona surperuana, la que albergaria a TIWANAKU, es laboratorio de un proceso de regionalización que implica términos de desarrollo y unidad geográfica. Nuestras investigaciones sobre la presencia TIWANAKU en el valle del Caplina demuestran fehacientemente que el antiguo Tiwanaku generó y engrosó diversos sistemas de unidad geográfica y cultural, que indudablemente pueden servir de referencia, mas no necesariamente de modelo o pauta al proceso que tiene lugar en nuestros dias.

La región, José Carlos Mariátegui, que admite a los departamentos de Tacna, Moquegua y Puno, reproduce la misma zona geográfica y cultural que habitó Tiwanaku en su oportunidad y abarcó asimismo, al norte chileno y noroeste boliviano. La simetria del espacio es obvia. Por tanto, Tiwanaku constituye en el tiempo, una de las identidades forjadas y actuantes en esta parte de América, y nos afecta directamente, porque no solamente es acervo sino presencia a través de los materiales arqueológicos encontrados y que lo reforzarán futuras investigaciones.

Tacna a través de su historia ha tenido sucesivas presencias culturales, siendo Tiwanaku una presencia con un espacio y tiempo definido. No obstante, en Tacna siempre ha estado vigente el problema de la identidad, por su situación económica y política. Ha sido punto de encuéntro de muchas identidades como ser tiwanaku, inka, aymara, española, africana, europea (inglesa, francesa, italiana), últimamente norteamericana, asiática, etc., sumándose hechos como la situación de Tacna como frontera con dos paises formados por los nacionalismos modernos y que antes de 1875 Tacna se constituye en el eje central de un vasto circuito comercial sudamericano, que englobaba espacios del norte chileno, norte argentino, oeste boliviano, sur peruano y sus conexiones con Panamá, Inglaterra. Esto hizo que Tacna fuese punto de encuentro de múltiples intereses económicos, detrás de los cuales existian diferentes y hasta contrapuestas identidades. En esa dimensión, es nuestro interés conocer el significado y los componentes de la presencia TIWANAKU en el valle del Caplina Tacna, cuál es el significado dentro del proceso histórico de Tacna, cómo interviene en este proceso y si aún podemos observar su impronta en su dinámica cultural.

\section{PRESENTACIÓN GENERAL}

En la zona sureste del lago Titicaca, hace más de 8 mil años existieron y se desarrollaron cazadores altoandinos que poseyeron una tradición litica denominada Viscachani.

Dichos grupos persistieron un periodo que se prolongó hasta aproximadamente los 2 mil años antes de nuestra era.

Aquellos cazadores evolucionan y fabrican cerámica. A esta fase se la denomina Chiripa, una expresión del Periódo Formativo del Altiplano peruano (Bennett: 1934:45). La cerámica Chiripa es similar a la cerámica Pukara de la zona noroeste del mismo lago y que es también una expresión formativa (Kidder:1945:10-26).

Se presume que la génesis de Tiwanaku se desarrolló paralelamente con las dos fases formativas del Altiplano (Chiripa, Pukara). La cerámica tiwanaku I tiene rasgos similares a la Chiripa. La Fase III estaria relacionada con la construcción del Templete semisubterráneo. La fase IV o Tiwanaku Clásico presenta una cerámica muy fina relacionada con el crecimiento de los asentamientos y el inicio del proceso tiwanaku propiamente. La fase $\mathrm{V}$ significó la expansión y evidenció la presencia tiwanaku en los valles occidentales y en las yungas orientales del área centrosurandina.

Diversos investigadores han levantado secuencias cronológicas para Tiwanaku (Bennett.1934; Wallace:1957). El primero introduce las fases clásica y decadente, que equivaldrian a las fases IV y V de Ponce Sanjinez. Sin embargo, esta clasificación discrimina a la cerámica fina y la tosca. En consecuencia, la $V$ seria la expansiva y la otra siempre se encontrará en los centros ceremoniales.

A la conocida secuencia de Tiwanaku se le ha dado una nueva nomenclatura de Estadios (Ponce S:1976;lbid:1991). Las épocas I y || corresponderian al Estadio Aldeano que desarrolló la agricultura, alfarería, pastoreo y un patrón aldeano. 
Las épocas III y IV corresponderian al Estadio Urbano. Se desarrolló la arquitectura ceremonial y un esquema urbanistico pronunciado (Ponce S:1991:7-27). En este Estadio tuvo vigencia el culto tiwanaquense. Es muy probable que fuera un momento de apogeo. El trabajo ceramista, metalurgista y otras manifestaciones culturales adquirieron categoría artística definida.

El Estadio Imperial o época $V$ supuso la expansión de Tiwanaku a la periferie, logrando una estructura de influencias bastante nitidas. No obstante, se apreció cierta declinación artística en relación al Estadio Urbano, épocas III y IV.

Es en el Estadio Imperial que Tiwanaku trama su hegemonia en prácticamente toda el área referida.

La expansión aludida se inicia aproximadamente a finales del siglo VIII y a comienzos del siglo IX de nuestra era, pero es probable que los primeros colonos fueran de la época IV (Goldstein:1990:30-31).

Tiwanaku al expandirse sitúa colonias lejos de su capital asegurándose una ecologia diferente y obteniendo productos que complementan su economia (Mujica:1983:85-102). (Brownman:1985:5971). La declinación de Tiwanaku es observada en los inicios del segundo milenio de nuestra era. En la zona circunlacustre emergen varios grupos postiwanaku con vocación independentista como también en todo el ámbito de la influencia tiwanaku, es asi que en los valles occidentales se manifiestan varios grupos que se 'emancipan' de Tiwanaku y evidencian ser de estirpe costeña, reforzando las identidades locales. Una nueva formación histórico social emerge en esta área andina.

La presencia tiwanaku puede hallarse en los valles de Moquegua, Locumba, Sama, Caplina, Azapa, Camarones y hasta en el oasis de San Pedro de Atacama. Los materiales arqueológicos demuestran una interacción del Altiplano y las poblaciones locales. Las relaciones altiplano-costa han merecido acuciosos estudios de investigadores como Murra (1975) y Rostworosky (1988), quienes advirtieron vínculos en periodos tardios.

Elías Mujica y colaboradores (1983:99-100) señalan que las relaciones de intercambio y complementariedad funcionaron desde etapas anteriores a los inkas. También se plantea que la reciprocidad, la complentariedad y los avances intensivos en la agricultura, como elementos que facilitaron la expansión y desarrollo de Tiwanaku a la periferie (Brownman: 1985:67), (Kolata:1985:30-31).
En su estadio imperial es cuando Tiwanaku se transforma en ciudad, se consolida y complejiza construyendo monumentos religiosos. El carácter de Imperio Tiwanaku se fundamenta en lo siguiente:

$1^{\circ}$ Una gran extensión de superficie, lo que asegura profundidad y dispersión, variedad y abundancia de recursos agricolas, ganaderos, mineros, existentes o en potencia, y climas diversos.

$2^{\circ}$ Una población bastante numerosa que revalorice su territorio y que forjen una identidad.

$3^{\circ}$ Una fuerte homogeneidad étnica, cultural e ideológica, para lograr la unidad e integración de su población en un espacio tan extenso.

$4^{\circ}$ Cierta autonomía económica, gracias a la variedad de recursos.

$5^{\circ} \mathrm{Gran}$ potencial tecnológico, penetración técnica, económica e ideológica.

Es en su estadio imperial cuando Tiwanaku penetra en los valles occidentales, donde encuentra a poblaciones que vivian de la agricultura y dentro de un patrón aldeano. Debió ser la compleja ideologia tiwanaquense, las innovaciones tecnológicas y económicas y por qué no la violencia, los elementos que intervienen en la construcción de Tiwanaku. Asi, las poblaciones de campesinos son anexadas a Tiwanaku, que va a tener una carácter de 'potencia' en los Andes, especificamente en el llamado Horizonte Medio, rol que comparte con WARI, que dominaba los Andes centrales.

La expansión Tiwanaku es un fenómeno de integración regional, pero cada valle tuvo su particular forma de responder a este proceso, considerando que las poblaciones que reciben a Tiwanaku eran de raigambre local y con incipientes conocimientos tecnológicos.

En el valle del Caplina-Tacna, las primeras referencias de la presencia tiwanaku son las de Max Uhle (1919), quien dio filiación cultural a una colección privada de materiales arqueológicos. Luego se tiene un vacío en las investigaciones respectivas hasta la década del 60, cuando Isabel Flores, Jesús Gordillo y Marko López, arqueólogos del Instituto Nacional de Cultura-Tacna, realizan investigaciones preliminares en base a materiales encontrados en los valles de Locumba, Sama y Caplina, estableciéndose la filiación pertinente con la Fase $V$ de Tiwanaku.

Nuestra investigación se basa en información proporcionada por el proyecto arqueológico de 
Tacna, (PAT), activado por el INC desde finales de la década del 80.

En todo momento, nuestro trabajo se basó en las siguientes hipótesis:

$1^{\circ}$ Que la presencia tiwanaku en el valle del Caplina tenga filiación cultural con la fase $\mathrm{V}$ altiplánica, $\mathrm{O}$ Fase Expansiva o Estadio Imperial.

$2^{\circ}$ La presencia Tiwanaku en el valle del Caplina constituye una expresión local, resultado de la expansión y de las interacciones culturales altiplánicas y locales.

$3^{\circ}$ Dar una cronologia relativa a la presencia Tiwanaku en el valle del Caplina correspondiente a los siglos IX y $\mathrm{X}$, de nuestra era, dentro del proceso de expansión señalado, guardando contemporaneidad con la presencia Tiwanaku en los valles vecinos.

$4^{\circ}$ Es probable que los asentamientos Tiwanaku en el valle del Caplina, tuvieran un aprovechamiento selectivo de los espacios, ubicándose en la parte media del valle, zonas de Magollo y Para.

$5^{\circ}$ La cerámica tiwanaku encontrada en el valle del Caplina podría manifestarnos la interacción de elementos culturales altiplánicos y locales. Se perciben el mantenimiento de forma, diseños, manufacturas, pero con un acabado y materias primas locales.

$6^{\circ}$ Que la presencia tiwanaku en el valle del Caplina significó el desarrollo de un proceso agroalfarero, con asentamientos relacionados a los campos de cultivo.

$7^{\circ}$ La presencia tiwanaku en el valle del Caplina puede considerarse como consecuencia de un proceso de integración regional, por la intensificación de las relaciones culturales y económicas con el Altiplano y los valles vecinos.

$8^{\circ}$ La presencia tiwanaku desarrolló intensivamente la agricultura en el valle del Caplina.

\section{MARCO GEOGRÁFICO}

\subsection{GEOMORFOLOGIA}

Los sitios tiwanaku en el valle del Caplina están ubicados en los sectores de Magollo y Para, parte media del valle.

La configuración de estos terrenos está dada por una pampa formada entre los cerros Caramolle, de mil msnm: al este de ios cerros
Molles y Chastudal de 400 y $600 \mathrm{msnm}$. La altitud promedio de la zona alcanza los $475 \mathrm{msnm}$. Los terrenos en cuestión se asientan sobre depósitos aluviales, originados por la acción erosiva del río Caplina. Los estamentos geológicos riolíticos están asentados sobre la formación Huaylillas (Jaén y Ortiz: 1963).

Caplina es una vocablo aymara que significa 'el que nunca llegará al mar'.

El primitivo nivel del río Caplina está dado por la cota de las pampas del Alto de la Alianza (770 msnm), es decir, la erosión ocasionó una diferencia de 210 metros en relación al nivel original.

El valle tiene un promedio de $4 \mathrm{~km}$ de ancho, agregándose unos $10 \mathrm{~km}$ más en las pampas de la Yarada y Hospicio, que es donde las aguas del Caplina fallecen, prolongándose el cono deyectivo del valle a partir de la zona de Magollo, ensanchándose todavía algo más hacia abajo, hasta llegar al mar, adoptando una figura triangular.

Los asentamientos tiwanaku se encuentran sobre terrenos planos y sobre depósitos aluviales designados Solonchak-órtico y Fluvisol-eútrico. Son tierras, óptimas para cultivos intensivos, siempre y cuando haya riego permanente. También en esta zona tenemos terrenos eriazos sin aptitud agropecuaria y forestal. Están cerca del cerro Arunta (ONERN:1976).

Actualmente, la zona es utilizada agricolamente mediante una irrigación moderna, con los escasos recursos hidricos del rioCaplina y el canal Uchusuma.

En términos generales, el terreno es plano con ligeras ondulaciones. Se tiene además, una gruesa capa de cantos rodados, cubierta por otra de sedimentos de utilidad en los cultivos establecidos.

\subsection{ECOLOGÍA}

Los sitios tiwanaku actualmente coexisten con cultivos de olivos y frutales. Magollo y Para poseen un potencial apreciable pero se reciente seriamente por la falta del recurso hídrico que padece Tacna desde siempre.

La ecología del valle fue estudiada por la ONERN. La zona de estudio pertenece al desierto subtropical, con una altitud promedio entre los 500 


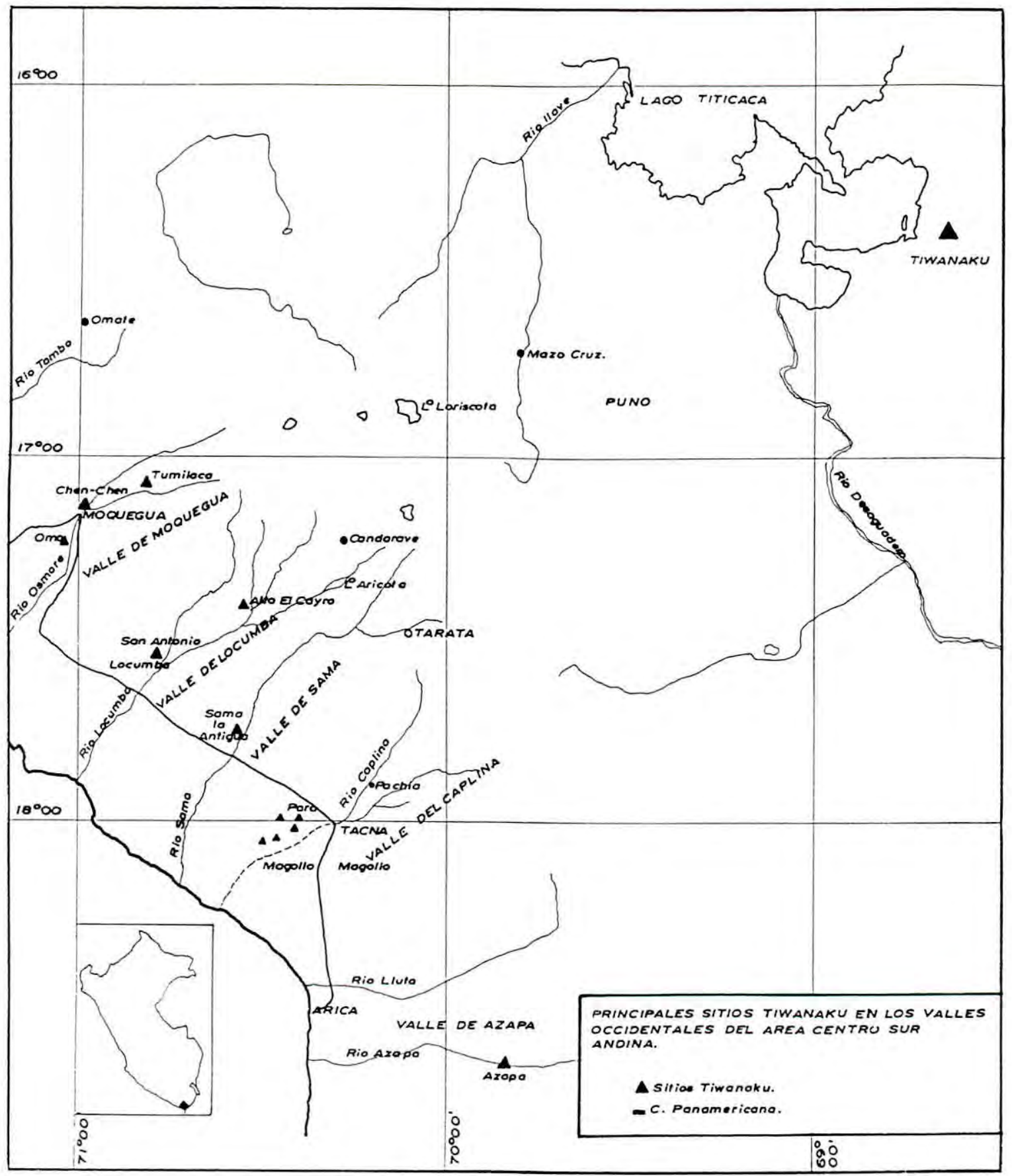

y 1500 msnm Como ya se dijo, actualmente se tienen alli cultivos de maíz (zea mais), ají (capsicum sp), zapallo (cucurbita sp), todos de origen prehispánico, además de frutales, olivares $y$ alfalfa.

En cuanto a árboles, tenemos el molle (lithra molle gay), chilcas (bacharis sp), gramas (triticum epin), más una variedad de árboles de procedencia europea como cipreses, eucaliptos y pinos. Igualmente puede registrarse la crianza moderada de ganado vacuno, ovino y porcino. En tiempos prehispánicos, el consumo de camélidos y cuyes constituyó dieta importantísima que hoy está absolutamente restringida.

\subsection{CLIMA}

Esta zona tiene clima cálido, árido y desértico. En los meses de invierno se presenta una fuerte humedad. Las precipitaciones pluviales se reducen a menos de $150 \mathrm{~mm}$ al año; sin embargo, la humedad y neblinas "camanchaca" en aymara), que se observan entre junio y agosto, cubren las planicies situadas entre los valles de Caplina y Sama, produciéndose una vegetación temporal, 
denominada Lomas, ecosistema que aportó en su momento a la economia una serie de recursos. Desde hace más o menos cincuenta años, las Lomas han venido desapareciendo hasta prácticamente reducirse a zonas de reserva.

\subsection{HIDROLOGIA}

En la parte media del valle confluyen los drenajes de los ríos Caplina, Caramolle (desaparecido) y el Uchusuma, canal artificial que aprovecha los excedentes del sistema Takora.

El río Caplina se origina en los deshielos de la cordillera del Barroso (5,742 msnm), que presenta glaciales que luego se convierten en bofedales y lagunas de regular dimensión.

El rio propiamente tal nace en la unión de las quebradas de Piscullane y Chillavane, desplazándose posteriormente en sentido noroeste y sur-oeste en su extensión de $109 \mathrm{~km}$.

El agua del Caplina es abundante en sales, sulfatos, posee un $\mathrm{PH}$ alto, es de sabor salobre y de moderada conductividad eléctrica. A tales aguas los especialistas denominan duras y son favorables para el cultivo de aji, pepinillos, zapallos y calabazas.

\section{SECUENCIA ARQUEOLÓGICA DEL VALLE DEL CAPLINA}

Recientes investigaciones han dado nuevas luces sobre el pasado del valle. En los año 60, Isabel Flores realiza exploraciones en casi todo Tacna y establece recurrencias entre los estilos postiwanaku de San Miguel, Pocoma y Gentilar (Flores:1969:295).

Las formulaciones de Uhle (1919) y Junius Bird quedaron obsoletas con la propuesta de Percy Dauelsberg (1973:23), ordenando los estilos postiwanaku en San Miguel y Gentilar.

Tenemos un vacio informativo para los periodos Arcaico hasta el Horizonte Medio (tiwanaku). En los valles de Moquegua y Azapa se encontraron vestigios de antiguas tradiciones de pescadores y recolectores como en el Ring Site (Richardson III et al :1990:139) que tiene una antigüedad de $10 \mathrm{mil}$ a 5 mil años antes de nuestra era. Este sitio ubicado en la costa de llo revela ser una de las "primeras adaptaciones marítimas en los andes" (sic).

En la cuenca del Osmore (Moquegua) se conoce el sitio arcaico de ASANA, donde cazadoresrecolectores vivieron entre los 9,500 y 3,500 ande
(Aldenderfer:1990:91-104).

Dicha ocupación arcaica parece mostrar las primeras evidencias de arquitectura en los Andes. Igualmente, serían pioneros en los procesos de sedentarismo y domesticación de camélidos.

Es pertinente mencionar a las cuevas Toquepala (Muelle:1969) (Ravinez:1967) y al abrigo de Caru (Ravinez:1967) como antecedentes arcaicos de Tacna. En la cueva de Toquepala se puede evidenciar varias ocupaciones, siendo la más antigua de 9,460 años de antigüedad. En Toquepala se ha definido una tradición lítica y se puede decir que se trataba de cazadores con cultura espiritual, pues la cueva $n^{\circ} 1$ fue lugar de actos propiciatorios para la caza.

Las primeras expresiones alfareras en los valles occidentales lo constituyen la cerámica de la fase HUARACANE de Moquegua, es cerámica con desgrasante vegetal (Feldman: 1990:227-236). (Ibid:1990 a: 65-74), y la cerámica ALTO RAMIREZ (Nuñez:1983:31-62), son las expresiones más tempranas de este valle. Tienen desgrasante vegetal y están asociadas al inicio de la agricultura.

En el valle del Caplina, últimamente, el PROYECTO DE CATASTRO ARQUEOLOGICO ubicó la cerámica 'EL ATAJO', la cual tiene desgrasante vegetal y con formas globulares con tratamiento y manufactura muy descuidada. Se ubica en el sitio del mismo nombre, en la parte final de Magollo, al oeste de los asentamientos Tiwanaku.

La cerámica El Atajo tiene mucha similitud con las expresiones alfareras de los valles de Moquegua y Azapa.

Aproximadamente en los inicios de la era cristiana, el poblamiento del valle se consolida. La población de El Atajo desarrolla ampliamente la agricultura. Cuando Tiwanaku inicia su expansión, aproximadamente siglo IX, las actividades agricolas se potenciarian significativamente, merced a la red de factores económico-culturales, altiplánicocosteños.

Las poblaciones tiwanaku asentadas en el valle del Caplina, zonas de Magollo y Para, igualmente practicaron la agricultura como medio de sustento básico.

No están claras las razones por las cuales declina Tiwanaku. En todas las áreas de influencia empezaron a surgir grupos locales con actitud independentista en relación al Estado tiwanaquense. En el valle del 
Caplina, los estilos postiwanaku: San Miguel, Pocoma, Gentilar, tienen evidencia en toda su extensión. Al parecer, en dicho periodo existió un poblamiento intensivo y extensivo. Sitios de aquel periodo son: Challatita (Santos:1980), Tocuco, Lluta(Trimborn:1975:15-18), Cristo Rey (Vela:1990:14142) y otros tantos que el proyecto Catástro Arqueológico viene descubriendo paulatina y reveladoramente.

La presencia inka parece haber tenido la participación de "mitimaes", que se establecen en la zona de Calientes y Pocollay, y que tienen origen altiplánico. En Peañas se ha encontrado evidencias del estilo inka, Chucuito, Saxamar, asociados a grupos locales como San Miguel, Pocoma, Gentilar y hasta el estilo Chuquibamba de Arequipa (Cavagnaro:1986) (Gordillo y García: 1989:10-25) expresándose el carácter multiétnico de su población.

\section{LA PRESENCIA TIWANAKU EN TACNA}

\subsection{PATRÓN DE ASENTAMIENTO TIWANAKU EN EL VALLE DEL CAPLINA}

En el valle del Caplina, las investigaciones realizadas por H.Trimborn en 1972, establecen que en el periodo intermedio tardío, las poblaciones del valle presentaban aldeas de terrazas domésticas (Trimborn:1975:39) en los sitios de Tocuco, Challatita, Lluta, en la parte media del valle.

Estando en la parte media del valle los asentamientos tiwanaku parece que aprovecharon selectivamente los espacios, al mismo tiempo, en tanto se ubicaron próximos a los drenajes, debieron utilizar muy racionalmente los recursos hidricos. En ello influye seguramente, el carácter de su organización social.

La disposición de los asentamientos tiwanakus en el valle del Caplina se manifiestan en pequeñas poblaciones de agricultores, en torno a los campos de ese uso, descubriéndose una directa vinculación entre los asentamientos y la producción.

Los sitios tiwanakus, ubicados en la zona de Magollo, manifiestan caracteristicas domésticas asociadas a campos de cultivo y canales de regadio.

Estos sitios demuestran contextos domésticos por la presencia de batanes, piedras de molienda, cerámica utilitaria, la misma que no presenta engobe y sus formas denotan funciones domésticas. Otros sitios adquieren funcionalidad complementaria a los asentamientos base, como los cementerios.

Es necesario indicar que antes de la presencia tiwanaku en el valle del Caplina, ya existía una población en el extremo de Magollo, que desarrollaba alfareria y agricultura y parece vinculada a un proceso Formativo. Este sitio. EI Atajo, de reciente hallazgo, fabricaba una cerámica con desgrasante vegetal, ésta tiene un fechado de 580 d.C., es un sitio doméstico ubicado en esta inmensa pampa, donde se observan varios sectores funcionales como unidades domésticas, geoglifos, zonas de actividad alfarera (Gordillo entrevista oral).

Se observa que cuando declina la presencia tiwanaku en los valles occidentales, sus asentamientos fueron reutilizados por las poblaciones de los desarrollos locales tardíos. En Azapa existen 24 sitios tiwanaku, reutilizados (Rivera: 1975), algo similar ocurre en Tumilaca, Moquegua (Bawden:1990:110).

Los sitios tiwanaku en el valle del Caplina evidencian una clara reocupación por los grupos San Miguel, Gentilar, etc.

En el periodo postiwanaku se realiza un poblamiento intensivo y extensivo en todo el valle, diferenciándose de ese modo de la presencia tiwanaku que restringió sus asentamientos a la parte media y final del valle.

Podemos mencionar a los sitios con evidencias tiwanaku:

El sitio Copare 4, el que tiene un cementerio y zona agricola. El sitio Magollo 2, es una sitio de caracteristica doméstica, donde se aprecia estructuras rectangulares de piedra de molienda, y de uso agrícola, en todo el sitio hay una gran recurrencia de materiales de Tiwanaku $\mathrm{V}$.

El sitio Para 1 es una cementerio muy disturbado, donde hay en su superficie fragmentos de cerámica tiwanaku, restos vegetales, maderas. Alli encontramos una jarrita de un "kero" de madera con aplicación burilada de diseño tiwanaku.

El sitio Cerro de Para 2 es una cementerio con dos concentraciones de tumbas de las mismas caracteristicas que el sitio Cerro de Para 1, ambos se separan entre sí por mil metros de distancia y 
están vinculados a las zonas de Magollo y Para. En su conjunto, ambos cementerios tienen aproximadamente 2 mil tumbas circulares y subterráneas, donde se encuentran cráneos deformados, restos de zapallos, maiz, esteras, conchas de marisco, espinas de pescado, huesos de camélido, fibras textiles.

\subsection{LA CERÁMICA TIWANAKU EN EL VALLE DEL CAPLINA}

En el valle del Caplina la cerámica con filiación tiwanaku fue descrita por Uhle en 1919. Posteriormente, son varios los investigadores que efectuaron estudios en la periferia tiwanaquense. Percy Dauelsberg, Mario Rivera, Guillermo Focacci e Iván Muñoz, investigadores chilenos, quienes en general coinciden, asegurando que las expresiones tiwanaku son consecuencia de manifestaciones entre tiwanaku (Altiplano) y las poblaciones locales. El planteamiento genérico formula que existe una cerámica denominada 'cabusa'.

En el valle del Caplina la presencia tiwanaku muestra una cerámica con evidente filiación con la fase $\mathrm{V}$ del Altiplano o expansiva imperial.

La cerámica altiplánica es más fina, con una textura más uniforme y su pasta es más homogénea, con una cocción más controlada, y no requiere de inclusiones como antiplástico, arena, mica o cuarzo (Girault: 1990:119-155).

La pasta de la cerámica tiwanaku del Caplina tiene cuarzo, arena y no es homogénea. La distribución de sus granos no es uniforme, la fractura es irregular y su cocción en general es oxidante, con algún descuido. No tiene muchas inclusiones.

El engobe suele ser de color rojo o rojo granate, parece haberse aplicado muy diluido. La cerámica utilitaria no presenta engobe y son básicamente ollas y cántaros de gran tamaño. Sus paredes son gruesas y sus formas y acabados burdos.

Podemos decir que no existe uniformidad en los aspectos tecnológicos y artísticos en la cerámica tiwanaku del valle del Caplina. Es categórico, sin embargo, que se trata de una cerámica local. En observaciones microscópicas, hemos podido apreciar que se usaron una variedad de arcillas, pero siempre dentro de un patrón estilistico.
No podemos decir que los cambios tecnológicos son indicadores correlativos de cambio y históricos pues la cerámica tiwanaku del Caplina, presenta variedad en cuanto a cocción, pasta, acabado, siendo fruto de una sociedad con contradicciones.

Con todo, la cerámica Tiwanaku en el Caplina es una expresión de la influencia tiwanaku, genera una serie de interacciones simbólicas y materiales, que representan una expresión tiwanaku local.

\subsubsection{Tipo I Vasos}

Este tipo es muy característico de la cerámica tiwanaku. Se trata de vasos de lados evertidos. La base denota diámetro más pequeño que la boca. Se les denomina también "keros". Ostentan engobe rojo y rojo granate. La decoración en negro sobre rojo, ocasionalmente con blanco. Exhiben una superficie lisa y pulida. La superficie anterior tiene un restregado horizontal, con engobe en los bordes.

Exhiben diseños geométricos de bandas horizontales y verticales: además, diseños o motivos escalonados. La pasta es fina o regularmente fina, compacta, de color naranja, rojo-naranja y de granos gruesos: tiene como desgrasante granos finos de cuarzo, el engobe parece que fue aplicado muy diluido. La cocción es oxidante, aunque no muy bien controlada.

\subsubsection{Tipo II Tazones}

Tipo muy similar a los vasos, pero difieren en el uso, pues los tazones fueron usados para beber liquidos y para ingerir alimentos. EI diámetro de la base es menor que el de la boca, pero la altura de sus lados es inferior al de los vasos. Estos tazones tienen engobe rojo o rojogranate, con motivos ornitomorfos (parihuanas). El tratamiento y su pasta es muy similar a la de los vasos.

\subsubsection{Tipo III Jarras}

Este tipo se halla conformado por jarras decoradas, globulares y de base plana. Muestran un asa que empieza en el cuerpo y termina antes del cuello. Superficie exterior pulida y con engobe, que suele tener diseños geométricos, escalerados, ondulados. La superficie interior tiene un restregado horizontal, con decoración en el borde. 


\subsubsection{Tipo IV Ollas y Cántaros}

Las ollas y cántaros constituyen un tipo que no presenta engobe y son cerámica utilitaria. Siempre se les ubica en contextos domésticos. Sus formas, tamaño y tratamiento concuerdan con su función. Estos ejemplares se encuentran muy erosionados por la falta de engobe. Su pasta es muy porosa, gruesa, con arena y cuarzo de desgrasante. La distribución de la pasta es homogénea, su fractura es irregular, tratamiento descuidado. La superficie exterior posee un restregado y bruñido horizontal. La superficie interior posee un restregado irregular. Los cántaros poseen unas falsas asas que son unos protúberos que se proyectan en el cuello. Las paredes de esta cerámica son más gruesas que la de los otros tipos. Tienen cocción oxidante, aunque no muy controlada. La pasta suele ser de color naranja-marrón y marrón. Se usaron para la elaboración y almacenaje de alimentos y líquidos.

\section{APROXIMACIÓN HISTÓRICA}

La presencia tiwanaku en el Caplina es el resultado de un proceso de integración que logró cohesionar la región, pero es cierto que cada valle tuvo su particular forma de responder a este proceso y dejaron su propia historia. Tiwanaku, en su capital, vivia en un complejo sistema de urbanismo, mientras en los valles costeños se encontraban pequeñas poblaciones de campesinos en condiciones precarias y en pequeñas aldeas. La introducción de tiwanaku debió combinar varios elementos innovadores a las poblaciones asimiladas, como el efecto de sus fundamentalismos. La evidencia arqueológica nos muestra una compleja parafernalia religiosa que en su momento fueron lugares que recibieron peregrinos de toda el área. Tiwanaku aporta con una innovación en la organización histórico-social en el área, como lo es el tránsito altiplano-costa, favorecido por la condición de migrante que posee el hombre andino.

Tiwanaku debió implantar sus patrones en la costa, los cuales asimilaron y se integraron a su sistema, por intercambio, reciprocidad y complementariedad con mecanismos comerciales. En esa medida, la economía de la zona va a ser más compleja y más dinámica, se establecen conexiones más rápidas y más eficientes entre poblaciones alejadas, por lo que se debió elevar la productividad en los valles. Esto impulsó el crecimiento demográfico, el valle del Caplina aumentó sus campos de cultivo como consecuencia de la presencia tiwanaku.

La religiosidad es un elemento superestructural en el hombre andino y su impronta hasta hoy la percibimos, pues la zona altiplánica mantiene dentro de su cosmovisión una compleja religiosidad con mucho contenido atávico y ancestral, siendo hasta hoy la zona circunlacustre el lugar de varios lugares que tienen carácter sagrado. Este influjo fue un elemento decisivo en este proceso. El investigador C. Ponce Sanginez (1991:25) plantea la posibilidad de que Tiwanaku mantuvo un ejército y que gracias a acciones militares se expandió hasta conformar un imperio.

No sabemos todavia la dimensión de la presencia tiwanaku dentro del contexto de las colonias tiwanaquenses y dentro de su geopolitica. Parece que Tiwanaku en el Caplina no tuvo la ingerencia y vínculos tan directos como lo fue con sus colonias en Moquegua (Goldstein:1990:31-58; 1990 a:75-114) Goldstein define 3 momentos para la ocupación Tiwanaku en Moquegua, un primer momento cuando los primeros colonos establecen contacto en el valle, esto lo relaciona con el Tiwanaku IV o Clásico (fase Omo). Luego se tiene la cerámica relacionada con Tiwanaku V (fase Chen-Chen) y una cerámica denominada Tumilaca que significa el final de la influencia tiwanaku en el valle y la introducción de elementos locales (fase Tumilaca).

Estamos convencidos que la presencia Tiwanaku se da tardiamente en el Caplina. Los materiales arqueológicos se relacionan con el Tiwanaku $\mathrm{V}$, comparativamente el valle del Caplina fue uno de los menos poblados, tal vez la escasez del recurso hídrico fue un factor agravante junto a la humedad y la configuración geográfica del Caplina, teniendo una producción agrícola limitada.

Cuando Tiwanaku llega al Caplina encuentra una población agroalfarera en 'El Atajo', quienes parece eran parte de lo que se denomina un Proceso Formativo. Hemos realizado exámenes comparativos de cerámica y apreciamos que la cerámica tiwanaku del Caplina, es tecnológicamente más avanzada, con respecto a la cerámica 'El Atajo'.

La expansión tiwanaku a los valles costeños logró asimilar estos territorios, y gracias a la arqueología se ha podido determinar el carácter regional de este proceso. En Moquegua se establece la fase Chen-Chen que significa la consolidación de Tiwanaku en ese valle (Goldstein:1990), como lo sería 
en Arica la fase Cabusa (Focacci:1990:69-124), (Muñoz:1983:43-114), aunque los materiales arqueológicos no guardan una estricta correlación, pues cada población tuvo su propia manera de responder a la influencia del Tiwanaku. En Tacna, especificamente en el valle del Caplina, proponemos la fase Magollo, la cual relaciona la presencia tiwanaku en este valle. Significa un momento de integración regional y de fuertes vínculos con el Altiplano. Esta fase la podemos ubicar gracias a materiales arqueológicos, además de los aspectos simbólicos que evidencian este momento de la historia.

Los límites culturales de la presencia tiwanaku en el valle del Caplina están dados, en su inicio, por el momento que Tiwanaku se instala en el valle, teniendo la presencia de la población de 'El Atajo', siendo el final de la presencia tiwanaku, cuando emergen poblaciones locales y de estirpe costeña (Maytas, Gentilar, San Miguel, Pocoma). Estos expresan una vocación independentista, pues parece que estos grupos tuvieron un carácter de señoríos. Estos grupos continuarán sus contactos con el Altiplano, donde también se da un proceso de desintegración del Tiwanaku, aunque no están claras las razones de la declinación de Tiwanaku, debió ser consecuencia de la agudización de sus contradicciones sociales y cuando las poblaciones de los valles van formando sus propias identidades, como también por la caducidad de la religión y la organización tiwanaquense.

Cuando acaba la influencia tiwanaku en el Caplina, se inicia un poblamiento intensivo y extensivo en todo el valle, desarrollando nuevos patrones culturales que consolidaron identidades locales. Es un desafio entender profundamente la presencia tiwanaku enTacna, es importante conocer las relaciones con los valles vecinos y una necesidad conocer la ruta de Tiwanaku, pues eso debió ser una elemento de dinámica cultural muy valioso, pues el tránsito al Altiplano estableció jornadas, puntos intermedios.
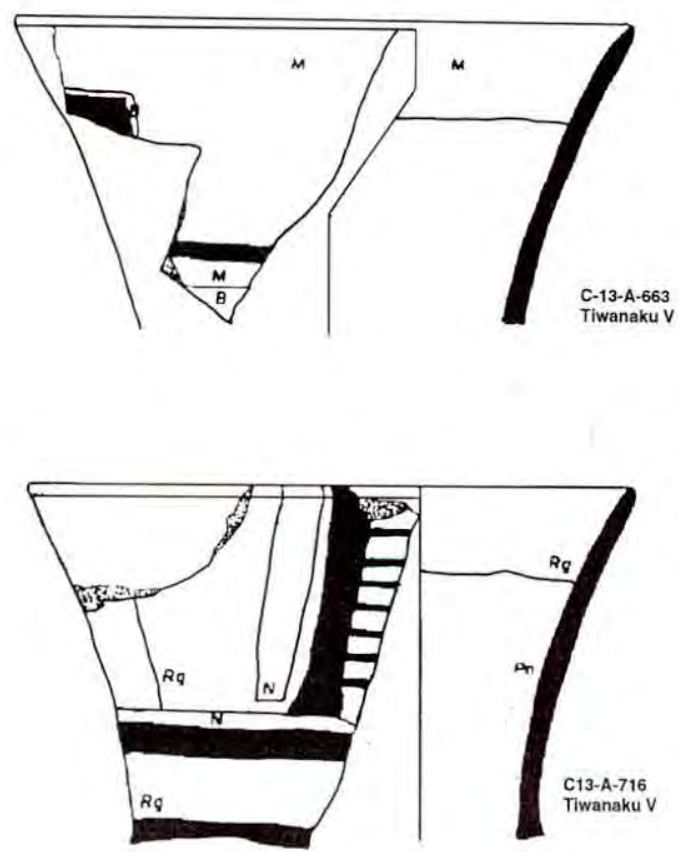
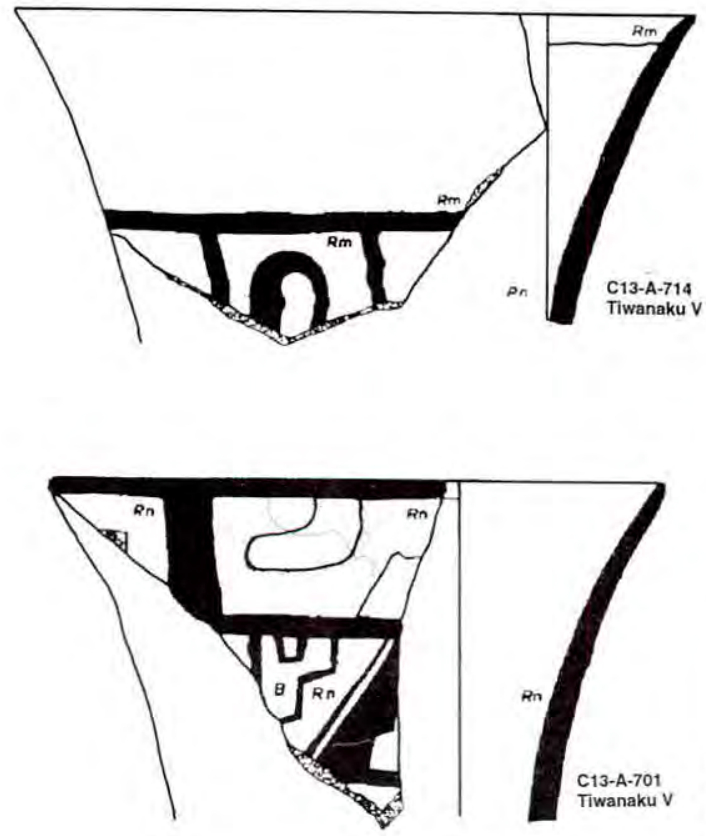
CERAMICA FASE MAGOLLO (TIPO 1 VASOS)
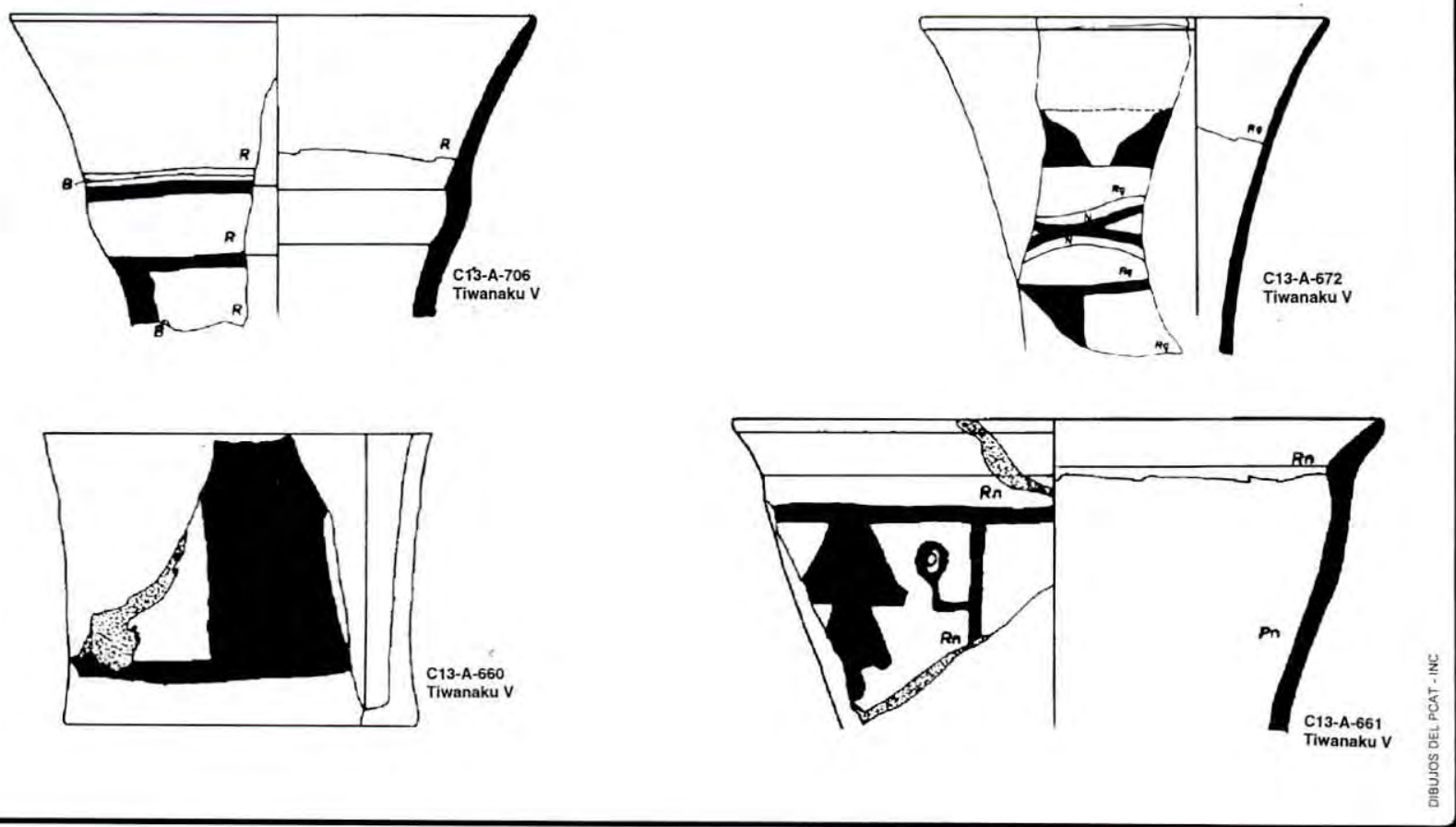

CERÁMICA FASE MAGOLLO (TIPO 2 TAZONES)
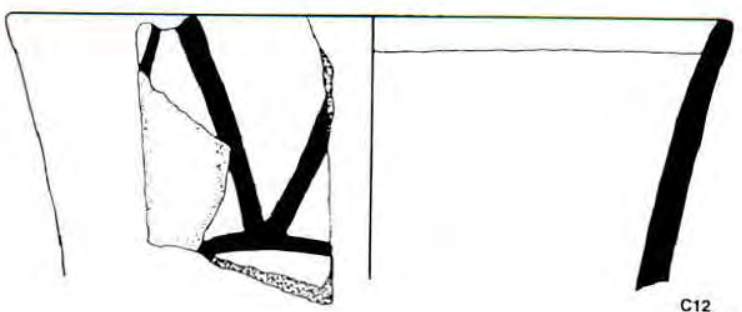

C12
Tiwanaku V
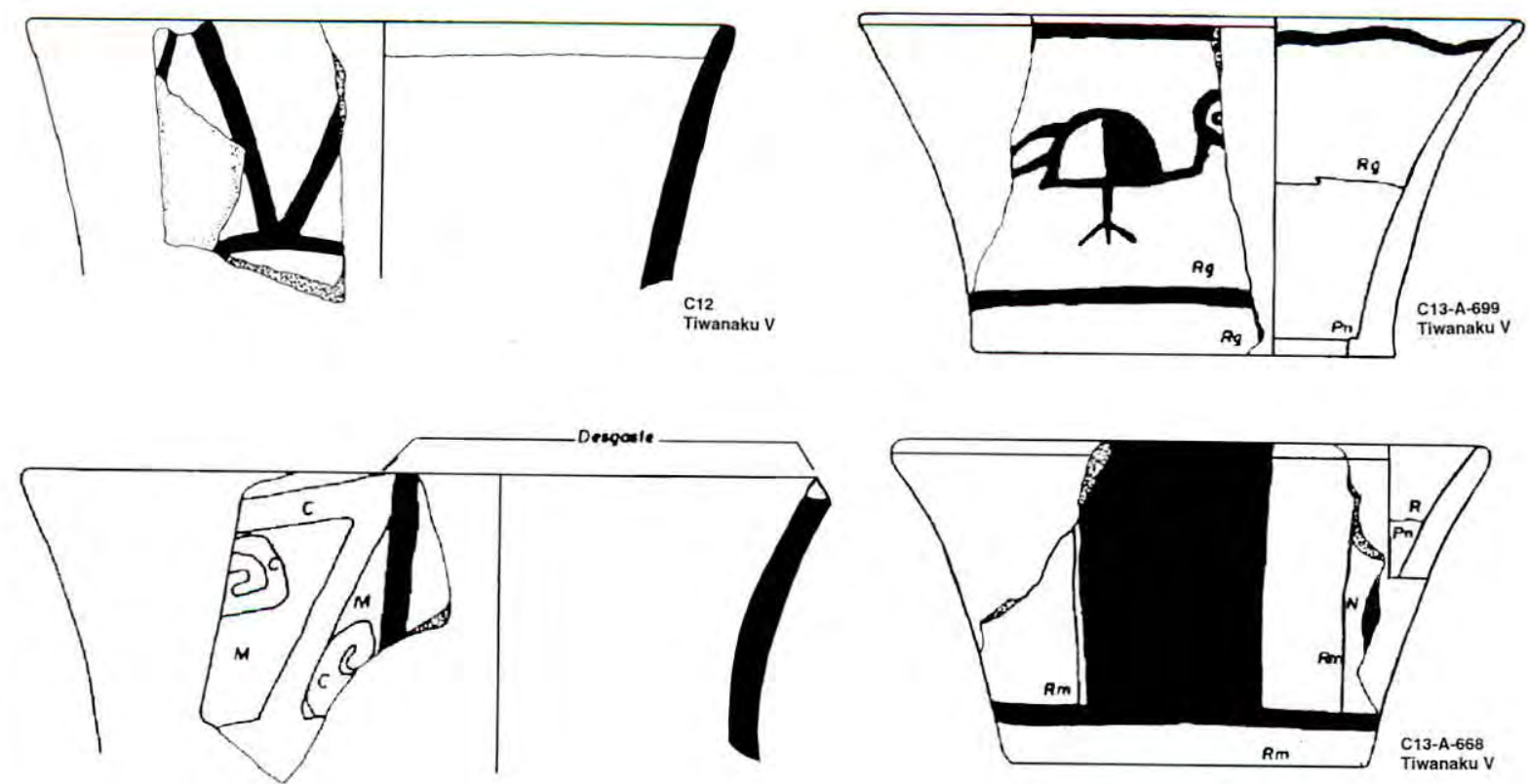

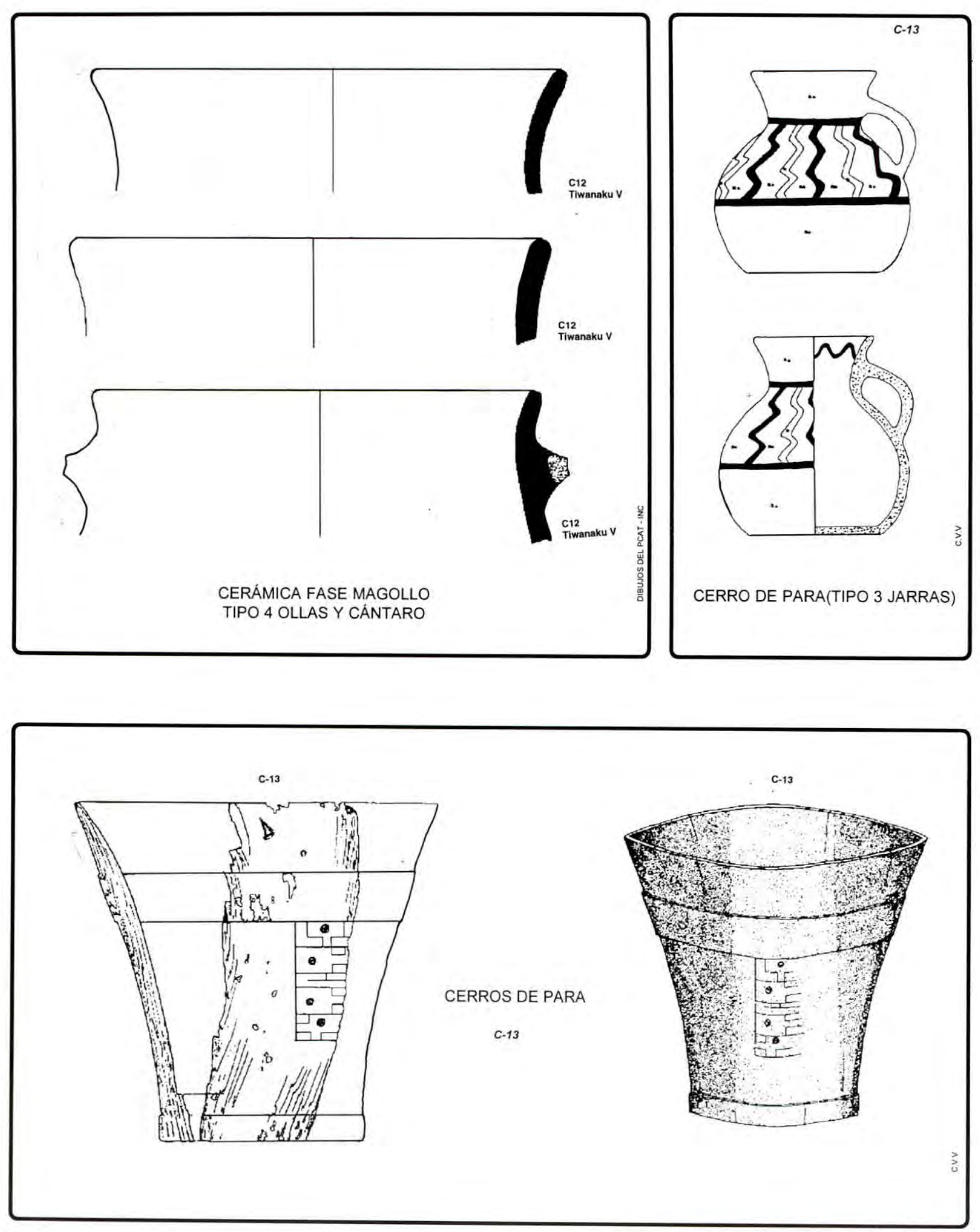


\section{BIBLIOGRAFIA}

\section{ALDENDERFER, Mark.}

1990. Asana: un yacimiento arcaico al aire libre en el sur del Perú. En trabajos Arqueológicos en Moquegua. Vol. 1. Edit. Escuela Nueva. Lima.

\section{BAWDEN, Garth.}

1990. El Sitio Tumilaca. En Trabajos Arqueológicos en MoqueguaPerú. Vol 2. pp 69-74. Watanabe et al Compiladores. Edit. Nueva Escuela, Lima.

BENNETT, Wendell.

1934. Excavations at Tiahuanaco. American Museum of Natural History. Anthropological Papers vol.34, part 3, New York.

BROWNMAN, David.

1985. Cultural Primacy of Tiwanaku in the development of later peruvian states. En Diálogo Andino $n^{\circ} 4$. Universidad de Tarapacá, Arica-Chile. Edit. Universitaria. Chile.

CAVAGNARO. Luis.

1986. Materiales para la Historia de Tacna. Tomo I. Edit. Cooperativa San Pedro de Tacna. Tacna.

DAUELSBERG, Percy.

1972. La Cerámica de Arica y su Situación Cronológica. En Chungara $n^{\circ} 1-2$. Universidad del Norte, Arica.

FOCACCI, Guillermo.

1990. Excavaciones arqueológicas en el cementerio AZ-6 Valle de Azapa. En Rev. Chungará $n^{\circ}$ 24/25 Universidad de Tarapacá. Arica. Edit. Universitaria.

FELDMAN, Robert.

1990. La cerámica del periodo Temprano de Moquegua. En Trabajos Arqueológicos en Moquegua, vol. 1. Edit. Escuela Nueva. Lima.

GIRAULT, Louis.

1990. La Cerámica del Templete Semi Subterráneo de Tiwanaku. Ediciones CERES. La Paz, Bolivia.

GOLDSTEIN, Paul.

1985. Tiwanaku Ceramics of The Moquegua Valley, Perú. Tesis de maestria. Dpto. de Antropologia, Universidad de Chicago.

1990a. La Cultura Tiwanaku y la relación de sus fases cerámicas en Moquegua en: Trabajos Arqueológicos en MoqueguaPerú. Vol 2,pp 31-58. Watanabe et al compiladores. Edit Nueva Escuela. Lima.

1990b. La Ocupación Tiwanaku en Moquegua. En Gaceta Arqueológica Andina $n^{\circ}$ 18-19. pp 75-104. Lima.

ISHIDA, Eiichiro.et al.

1960. Andes. Report of the University of Tokio Scientific Expedition to the Andes in 1958. Tokio,Bijitsu Shuppan sa.

JAEN, Hugo y ORTIZ, Guillermo.

1963. Geología de los Cuadrángulos de la Yarada y Tacna. Edit. Carta Geológica Nacional. Lima.

KOLATA, Alan.

1985. El papel de la Agricultura Intensiva en la Economía Política del Estado Tiwanaku. En Dialogo Andino $n^{\circ} 4$, Dpto. de Historia y Geografia. Universidad de Tarapacá,Arica.
KIDDER, Alfred.

1943. Some early sites in the Norther Lake Titicaca Basin. Papers Peabody Museum of American Archaelogy and Ethnology. Harvard University, vol. $27 n^{\circ} 1$. Cambridge.

LUMBRERAS, Luis.

1981. La Arqueologia como Ciencia Social. Edit. PEISA. Lima.

MEGGERS, Betty y EVANS, Clifford.

1969. Cómo interpretar el Lenguaje de los Tiestos. Manual para Arqueólogos. Smithsonian Institution. Washington D.C.

MUJICA, Elias. RIVERA, Mario. LYNCH, Thomas.

1983. Proyecto de Estudio sobre la Complementariedad económica Tiwanaku en los valles occidentales del Area Centro-Sur Andina. En Chungará $n^{\circ} 11$. Universidad de Tarapacá.Arica.

MURRA, John.

1975. Formaciones Económicas y Políticas en el Mundo Andino. Instituto de Estudios Peruanos. Lima.

MUÑOZ, Iván.

1983. El poblamiento Aldeano en el valle de Azapa y su vinculación con Tiwanaku (Arica-Chile). En Documentos de Trabajo $n^{\circ} 3 p p$ 43-114. Universidad de Tarapacá Arica-Chile.

NUN̄EZ, Lautaro.

1983. Cerámica Temprana en Cáñamo (costa desértica del norte de Chile): Análisis y evaluación regional. En Chungará $n^{\circ} 11$. Universidad de Tarapacá. Arica. Edit. Universitaria. Chile.

O.N.E.R.N.

1976. Inventario, Evaluación y uso racional de los recursos naturales de la costa: cuenca de los ríos Moquegua, Locumba, Sama y Caplina. ONERN publicación $n^{\circ} 43$. Lima.

PONCE S., Carlos.

1972. Tiwanaku: espacio, Tiempo y Cultura. Ensayo de sintesis arqueológica. Academia Nacional de Ciencias de Bolivia. $N^{\circ} 30$. La Paz.

PONCE S. Carlos.

1991. El Urbanismo de Tiwanaku. en PUMAPUNKU, año $1, n^{\circ} 1$, nueva época. Producciones CIMA. La Paz-Bolivia.

TRIMBORN, Hermann.

1975. Investigaciones Arqueológicas en los valles de Caplina y Sama. Edit. Verbo Divino Estella, Navarra-España.

UHLE, Max.

1919. La Arqueología de Arica y Tacna. Imp. Universidad Central, Quito Ecuador.

VELA, Carlos.

1990. Estudio de los contextos funerarios del cementerio prehispánico Cristo Rey-Tacna. Tesis de bachiller en Arqueologia, Universidad Católica Santa Maria, Arequipa.

1992. Tiwanaku en el valle del Caplina. En PUMAPUNKU, año 1 , número 3, nueva época. Producciones CIMA. La Paz-Bolivia.

WATANABE, Luis y STANISH, Charles.

1990. Ocupaciones domésticas en el periodo Tiwanaku Tardio. Otora, Moquegua. En Trabajos Arqueológicos en Moquegua- Perú. Vol. 2, pp 75-96. Edit. Nueva Escuela. Lima. 